\title{
Dose study of electrocardiogram automatic tube current modulation technology in prospective coronary computed tomography angiography scans of overweight patients
}

\author{
GUIRU HE, XIAOPEI LIU, YAN LIU, WEI WANG and ZHILIANG KE
}

CT/MR Room, The Fifth Affiliated Hospital of Xinjiang Medical University, Ürümqi, Xinjiang 830000, P.R. China

Received September 9, 2014; Accepted March 6, 2015

DOI: $10.3892 /$ etm.2015.2412

\begin{abstract}
The aim of the present study was to investigate the X-ray absorption dose and clinical applications of electrocardiogram (ECG)-gated automatic tube current modulation (ATCM) technology in prospective sequential computed tomography coronary angiography (CTCA) scans of overweight patients. A total of 40 patients with a body mass index of $>24$ were divided at random into groups A and B. Group A consisted of 20 patients, who were examined using ECG-ATCM scanning. For the patients in group A with heart rates $<70 \mathrm{bpm}$, the scanning range was $20-80 \%$ of the R-R interval and the patients received full-dose X-rays for $60-80 \%$ of the R-R interval. For the group A patients with heart rates $>70 \mathrm{bpm}$, the scanning range was $20-80 \%$ of the R-R interval and the patients received full-dose scanning for $35-55 \%$ of the R-R interval. For the 20 patients in group B, the scanning range was $20-80 \%$ of the $\mathrm{R}-\mathrm{R}$ interval and patients received a full dose of X-ray radiation for the entire scanning period. The image quality and radiation dose was compared between the two groups. The average radiation dose in groups A and B was $6.91 \pm 2.78$ and $10.43 \pm 3.36$, respectively. The radiation doses in group A were reduced by $33.77 \%$ when compared with group $\mathrm{B}(\mathrm{P}<0.05)$. However, there was no marked difference observed in image quality. In summary, using ECG-ATCM technology in prospective sequential CTCA scanning may significantly reduce the radiation dose required for overweight patients.
\end{abstract}

\section{Introduction}

At present, there are two scanning modes for computed tomography coronary angiography (CTCA); prospective

Correspondence to: Dr Guiru He, CT/MR Room, The Fifth Affiliated Hospital of Xinjiang Medical University, 118 Henan West Road, Ürümqi, Xinjiang 830000, P.R. China E-mail: guiruhe@126.com

Key words: dual-source computed tomography, low dose, coronary artery, electrocardiogram automatic tube current modulation technique, sequential scanning, overweight and retrospective electrocardiogram (ECG)-gated scanning. Retrospective ECG-gated spiral scanning requires higher doses of radiation than prospective scanning $(1,2)$. Prospective sequential scanning is able to effectively reduce the radiation dose required for a narrow scanning window, which is increasingly used in CTCA examinations (3-5). However, research is mostly focused on low-dose adaptive sequential sequencing (6). There are a limited number of studies that investigated how radiation doses may be optimized according to changes in the heart rate in ECG pulsing windows of prospective sequential CTCA scanning. Thus, further research is required into methods of reducing the radiation dose of a CTCA scan, while ensuring the image quality of the coronary artery remains sufficient. This is particularly important for overweight [body mass index (BMI) of $>24$ ] patients who typically require higher radiation doses in order to obtain high quality images. In the present study, overweight patients were examined using ECG-gated automatic tube current modulation (ATCM) technology in prospective sequential scanning. The dose and exposure times were adjusted according to changes in the heart rate, and the feasibility of the radiation dose and imaging of the coronary arteries were compared.

\section{Materials and methods}

Patients. Between March and August 2013, patients suspected of having coronary heart disease were recruited to undergo a CTCA examination in the Fifth Affiliated Hospital of Xinjiang Medical University (Ürümqi, China). A total of 40 patients were recruited, of which 12 were female and 28 were male. Patient age ranged between 32 and 65 years, with an average age of 55.6 years. The BMI scores ranged between 25.6 and 30 . Patients were divided randomly into two groups. Group A consisted of 20 patients, 14 male and 6 female, with an age range of 32-65 years (mean age, 52.8 years) and a mean BMI of $27.8 \pm 2.84$. For group A patients with heart rates $<70 \mathrm{bpm}$, the scanning range was $20-80 \% \mathrm{R}-\mathrm{R}$ interval and the patients received a full dose of X-rays for $60-80 \%$ of the R-R interval. In the group A patients with heart rates $>70 \mathrm{bpm}$, the scanning range was $20-80 \% \mathrm{R}-\mathrm{R}$ interval and the patients received a full radiation dose for $35-55 \%$ of the R-R interval. For group $B$ patients (male, 17; female, 3; age range, 33-62 years; average age, 57.6 years; average BMI, 28.08 \pm 1.96 ) with any heart rate, the scanning range was $20-80 \%$ of the $\mathrm{R}-\mathrm{R}$ interval and 
Table I. Comparison of statistical results of the image quality between groups A and B.

\begin{tabular}{|c|c|c|c|c|c|}
\hline Group & $\mathrm{n}$ & $\mathrm{BMI}\left(\mathrm{kg} / \mathrm{m}^{2}\right)$ & CTDIvol (mGy) & $\operatorname{DLP}(\mathrm{mGy} \cdot \mathrm{cm})$ & $\mathrm{ED}(\mathrm{mSv})$ \\
\hline A & 20 & $27.8 \pm 2.84$ & $28.82 \pm 18.00$ & $406.2 \pm 163.3$ & $6.91 \pm 2.78$ \\
\hline B & 20 & $28.08 \pm 1.96$ & $51.86 \pm 10.63$ & $613.3 \pm 197.5$ & $10.43 \pm 3.36$ \\
\hline Value $\mathrm{t}$ or $\mathrm{t}^{\prime}$ & N/A & 1.159 & 8.975 & 7.575 & 7.575 \\
\hline P-value & N/A & 0.249 & 0.000 & 0.000 & 0.000 \\
\hline
\end{tabular}

Results are expressed at the mean \pm standard deviation. Group A, utility optimization technology group; group B, standard technology group; BMI, body mass index; CTDIvol, computed tomography dose index volume; DLP, dose-length product; ED, effective dose.

patients received full-dose radiation for the entire scan. The two groups presented stable heart rates prior to examination. Image quality and radiation dose were compared between the two groups.

Patients provided written consent prior to all procedures. Patients with severe liver/kidney dysfunction, allergy to iodine contrast agent, decompensated cardiac insufficiency or those that were unable to hold their breath were excluded from the study.

Equipment and scanning method. Patients underwent respiratory training prior to scanning of the entire heart region by ECG-gated detection, using a second-generation dual-source computed tomography scanner (SOMATOM Definition Flash; Siemens Healthcare, Munich, Germany). Monitoring levels were set at $1-2 \mathrm{~cm}$ under the trachea bifurcation, and the region of interest was selected in the aortic arch. Bolus tracking was used to initiate the automatic scan once the trigger threshold of the $180 \mathrm{HU}$ was reached (delay, $8 \mathrm{sec}$ ). An injection of 70-90 $\mathrm{ml}$ iopamidol (iodine content, $370 \mathrm{mg} / \mathrm{ml}$; Shanghai Bracco Sine Pharmaceutical Corp. Ltd., Shanghai, China) was administered at a rate of $6 \mathrm{ml} / \mathrm{sec}$. Scanning parameters were as follows: Tube rotation speed, $0.28 \mathrm{r} / \mathrm{sec}$; detector area, $128 \times 0.6 \mathrm{~mm}$; voltage, $120 \mathrm{kV}$; reconstruction interval, $0.5 \mathrm{~mm}$; and reconstruction thickness, $0.75 \mathrm{~mm}$. The tube current-time product was automatically set according to the patient BMI.

Image quality analysis. Image data were postprocessed by two experienced physicians using a double-blind method. Coronary arteries were divided into 15 segments, following the improved coronary artery segmentation techniques of the American Heart Association (7). The image quality evaluation standards were divided into three grades as follows: Grade I, coronary artery has no artifacts and the contour is clear; grade II, local coronary arteries have artifacts or vasculature is unclear; and grade III, coronary vascular outline is unclear, or the middle segment or the majority of the segments have artifacts. Grade I and II images were sufficient for evaluation and diagnosis, while grade III images were of insufficient quality.

Calculation of $X$-ray radiation dose. The method used for calculating the effective $X$-ray radiation dose of the CTCA scans was as follows (8): Effective radiation dose (ED) = dose-length product (DLP) $\mathrm{x}$ conversion coefficient of the examined position (K). For example, $\mathrm{ED}=\mathrm{DLP}(\mathrm{mGy} \cdot \mathrm{cm}) \times 0.017[\mathrm{mSv} /(\mathrm{mGy} \cdot \mathrm{cm})]$, when chest conversion coefficient is $\mathrm{K}=0.017[\mathrm{mSv} /(\mathrm{mGy} \cdot \mathrm{cm})]$.
Statistical analysis. Using SPSS software, version 13.0 (SPSS, Inc., Chicago, IL, USA), the $\chi^{2}$ test was conducted for image quality evaluation of the coronary arteries and the t-test was used for the two independent samples. $\mathrm{P}<0.05$ was considered to indicate a statistically significant difference.

\section{Results}

General patient data. Patients underwent a routine sequence examination in addition to optimized ECG tube-current modulation. No adverse reactions were observed. The BMI values in groups A and B were $27.8 \pm 2.84$ and $28.08 \pm 1.96 \mathrm{~kg} / \mathrm{m}^{2}$, respectively, and no significant difference was observed between the groups ( $\mathrm{P}>0.05$; Table I).

Image quality analysis. There were 505 and 494 coronary artery segments in groups $\mathrm{A}$ and $\mathrm{B}$, respectively. The number of patients with an image quality score of grade I in groups A and B was 406/505 (80.4\%) and 391/494 (79.1\%), respectively. In total, 81/505 (16.0\%) patients in group A and 82/494 (16.6\%) patients in group B received an image quality score of grade II. With a grade III image quality score, there were 18/505 (3.6\%) patients in group A and 21/494 (4.3\%) in group B, and no significant difference was identified between the groups. The assessment rates of groups A and B were 96.4 and $95.7 \%$, respectively ( $\mathrm{P}=0.57$; Table II). Grade III-quality images were frequently acquired from the middle and distal artery segments. A single patient in group A exhibited heart rate fluctuations that caused the scanning acquisition period to fall out of the full-dose exposure area, increasing image noise. Although the phase was reconstructed, vessel artifacts appeared.

Calculation of the $X$-ray radiation dose. In group A patients with heart rates $<70 \mathrm{bpm}$ (Fig. 1), the scanning range was $20-80 \%$ of the R-R interval and patients received full-dose radiation for $60-80 \%$ of the R-R interval. For group A patients with heart rates $>70 \mathrm{bpm}$ (Fig. 2), the scanning range was $20-80 \%$ of the R-R interval and patients received full-dose radiation for $35-55 \%$ of the $\mathrm{R}-\mathrm{R}$ interval. In group $\mathrm{A}$, patients received an X-ray dose that was $20 \%$ of the full dose for $20-35 \%$ and $55-80 \%$ of the R-R interval. In group B, the scanning range was $20-80 \%$ of the R-R interval and patients received a full radiation dose for the entire scan (Fig. 3). In the present study, the computed tomography dose index volume (CTDIvol) of group A was $55.57 \%$ of that in group B, and the DLP and ED were 66.23 and $66.25 \%$ of the group B values, 
Table II. Assessment of image quality for coronary artery CTA segments in groups A and B.

\begin{tabular}{|c|c|c|c|c|c|c|c|c|c|c|}
\hline \multirow[b]{2}{*}{ Coronary artery segments } & \multicolumn{3}{|c|}{ A } & \multicolumn{3}{|c|}{ B } & \multicolumn{2}{|c|}{ Total } & \multicolumn{2}{|c|}{$\begin{array}{c}\text { Assessment } \\
\text { Ratio (\%) }\end{array}$} \\
\hline & I & II & III & I & II & III & $\mathrm{A}$ & B & A & B \\
\hline RCA (proximal) & 33 & 7 & 0 & 37 & 3 & 0 & 40 & 40 & 100 & 100 \\
\hline RCA (middle) & 29 & 8 & 3 & 30 & 8 & 2 & 40 & 40 & 92.5 & 95 \\
\hline RCA (distal) & 28 & 7 & 2 & 31 & 6 & 2 & 37 & 39 & 94.6 & 94.9 \\
\hline PDA (right) & 19 & 9 & 0 & 19 & 10 & 1 & 28 & 30 & 100 & 96.7 \\
\hline LM & 37 & 3 & 0 & 37 & 3 & 0 & 40 & 40 & 100 & 100 \\
\hline LAD (proximal) & 36 & 4 & 0 & 35 & 5 & 0 & 40 & 40 & 100 & 100 \\
\hline LAD (middle) & 34 & 4 & 2 & 32 & 6 & 2 & 40 & 40 & 95 & 95 \\
\hline LAD (distal) & 23 & 12 & 2 & 28 & 9 & 3 & 37 & 40 & 94.6 & 92.5 \\
\hline D1 & 29 & 7 & 2 & 25 & 8 & 1 & 38 & 34 & 94.7 & 97.1 \\
\hline D2 & 13 & 2 & 2 & 8 & 3 & 1 & 17 & 12 & 88.2 & 91.7 \\
\hline LCX (proximal) & 37 & 3 & 0 & 33 & 7 & 0 & 40 & 40 & 100 & 100 \\
\hline LCX (distal) & 36 & 2 & 1 & 29 & 4 & 3 & 40 & 36 & 97.5 & 91.7 \\
\hline $\mathrm{OM}$ & 27 & 6 & 2 & 30 & 4 & 3 & 35 & 37 & 94.3 & 91.9 \\
\hline PL & 21 & 6 & 2 & 16 & 5 & 3 & 29 & 26 & 93.1 & 88.5 \\
\hline PDA (left) & 4 & 1 & 0 & 1 & 1 & 0 & 5 & 2 & 100 & 100 \\
\hline Total & 406 & 81 & 18 & 391 & 82 & 21 & 506 & 496 & 96.3 & 96.4 \\
\hline$\chi^{2}$ & & & & & & & & & & \\
\hline
\end{tabular}

CTA, computed tomography angiography; A/B, group A/B; I/II/III, grade I/II/III; RCA, right coronary artery; PDA (right/left), posterior descending artery (right/left dominant type); LM, left main; LAD, left anterior descending; D, diagonal; LCX, left circumflex; OM, obtuse marginal; PL, posterior left.

respectively (Table I). No significant difference in image quality was observed between the groups. For patients with a high BMI, selecting a different total dose exposure window according to different heart rates appeared to reduce the required radiation dose.

In group A, the CTDIvol was $28.82 \pm 18.00 \mathrm{mGy}$, the DLP was $406.2 \pm 163.3 \mathrm{mGy} \cdot \mathrm{cm}$ and the average ED of radiation was $6.91 \pm 2.78 \mathrm{mSv}$. In group B, the CTDIvol was $51.86 \pm 10.63 \mathrm{mGy}$, the DLP was $613.3 \pm 197.5 \mathrm{mGy} \cdot \mathrm{cm}$ and the average ED of radiation was $10.43 \pm 3.36 \mathrm{mSv}$. Significant differences in CTDIvol, ED, DLP and ED average were observed between the groups $(\mathrm{P}<0.05)$. The assessment of image quality for coronary artery CTA segments indicated that there was no significant difference in image quality between the groups.

\section{Discussion}

CTCA imaging is used widely in clinical practice, however high radiation doses are potentially harmful and their effects are under increasing investigation (9). The American Food and Drug Administration Commission issued a statement by the American Heart Committee, stating that a $10 \mathrm{mSv}$ dose of CT radiation may cause $1 / 2,000$ patients to develop a malignant tumor (10). Davis et al (11) demonstrated that a higher radiation dose increased the risk of testicular cancer and glioma in patients that underwent CT scans. Therefore, doctors and imaging technicians aim to maintain the quality of coronary artery imaging during CTCA scanning, whilst also limiting the harm caused to the body by excessive X-ray radiation. It is important to design optimized scanning procedures in order to ensure that high quality coronary artery images are obtained using the lowest possible radiation dose. Current methods of controlling dual-source CT radiation dose include automatic mA modulation, variable and intelligent filtering technology, prospective gating control scan mode and phase-selective exposure technology. A report by the American Heart Association concluded that gating scanning technology was the most promising of these technologies (12).

BMI is the internationally recognized system for assessing the degree of obesity in overweight patients. In the case of obese patients, the required X-ray penetration increases with the volume of subcutaneous fat and muscle thickness, therefore the necessary radiation dose may also increase (13). The smaller the radiation dose, the worse the image quality. When BMI is high, an increased tube current is required to ensure image quality (14). However, by using tube current modulation in dual-source CT scanning, the tube current may be automatically adjusted as required, which reduces the X-ray radiation dose administered to patients. In the present study, varying tube currents were administered at different ECG periods. The radiation dose administered in group A following optimization $(6.91 \pm 2.78)$ was significantly lower when compared with group B (10.43 \pm 3.36$)$ without optimization, with no significant difference in image quality (Figs. 1-3).

Arnoldi et al (15) demonstrated that when using prospective scanning, the required radiation dose is $2.8 \mathrm{mSv}$, which is markedly lower than the retrospective scanning dose of $18.4 \mathrm{mSv}$. A prospective series scan is a step-axis scanning mode, 


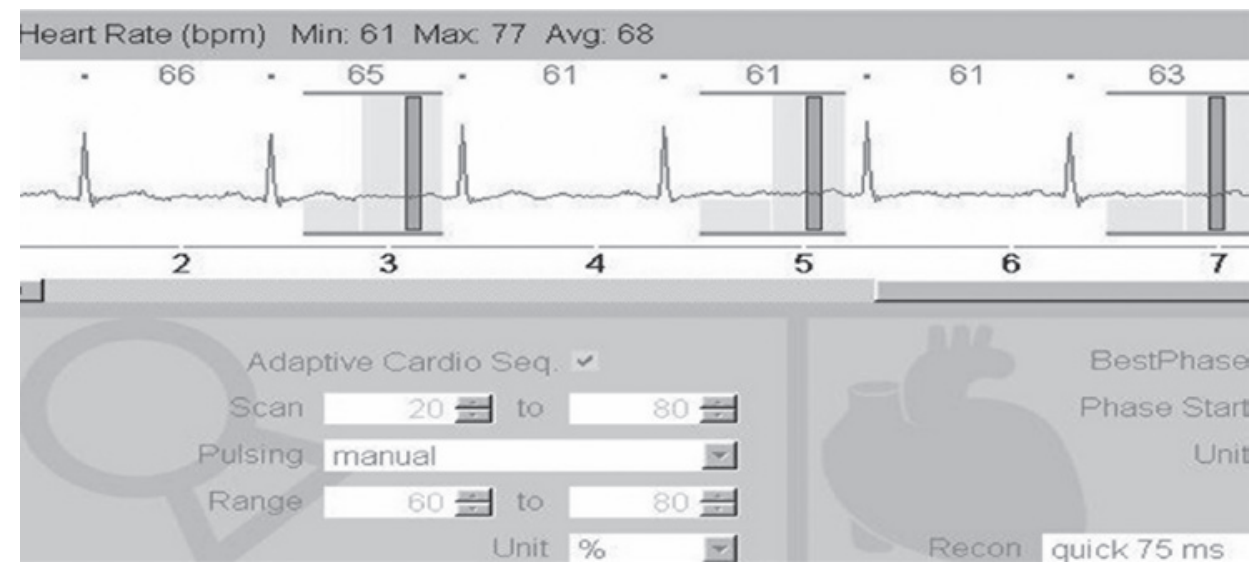

Figure 1. For group A patients with heart rates $<70 \mathrm{bpm}$, the scanning range was $20-80 \%$ of the R-R interval, with patients receiving full-dose scanning for $60-80 \%$ of the R-R interval.

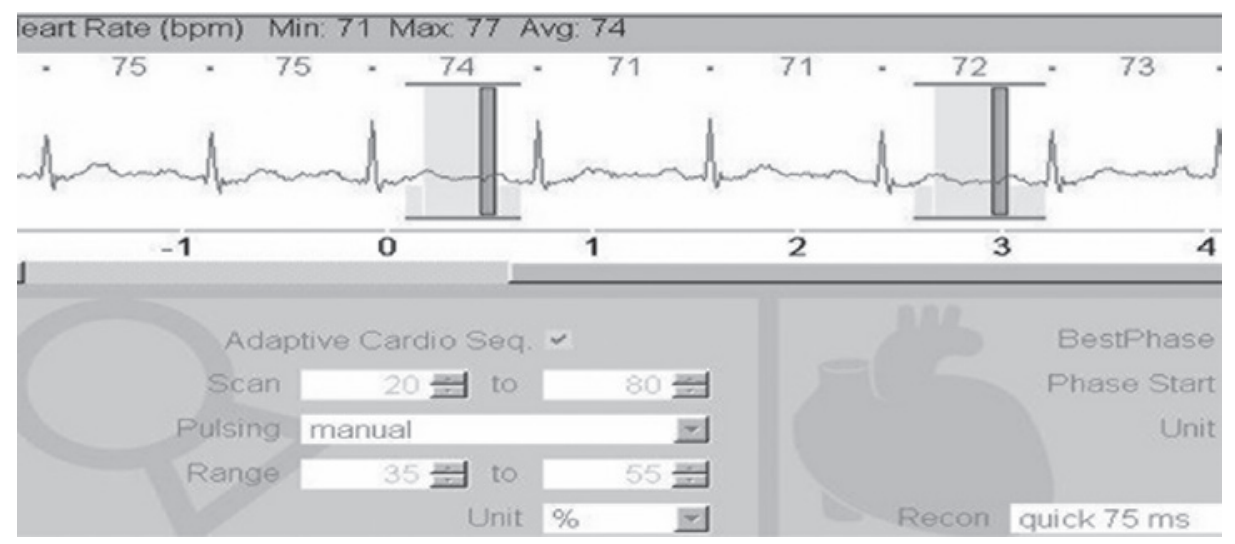

Figure 2. Image quality of coronary artery echocardiogram. For group A patients with heart rates $>70 \mathrm{bpm}$, the scanning range was $20-80 \%$ of the R-R interval and patients received a full X-ray dose for $35-55 \%$ of the R-R interval. Scanning sketch diagram and multiplanar reconstruction map of scanning range of $20-80 \%$ interval.

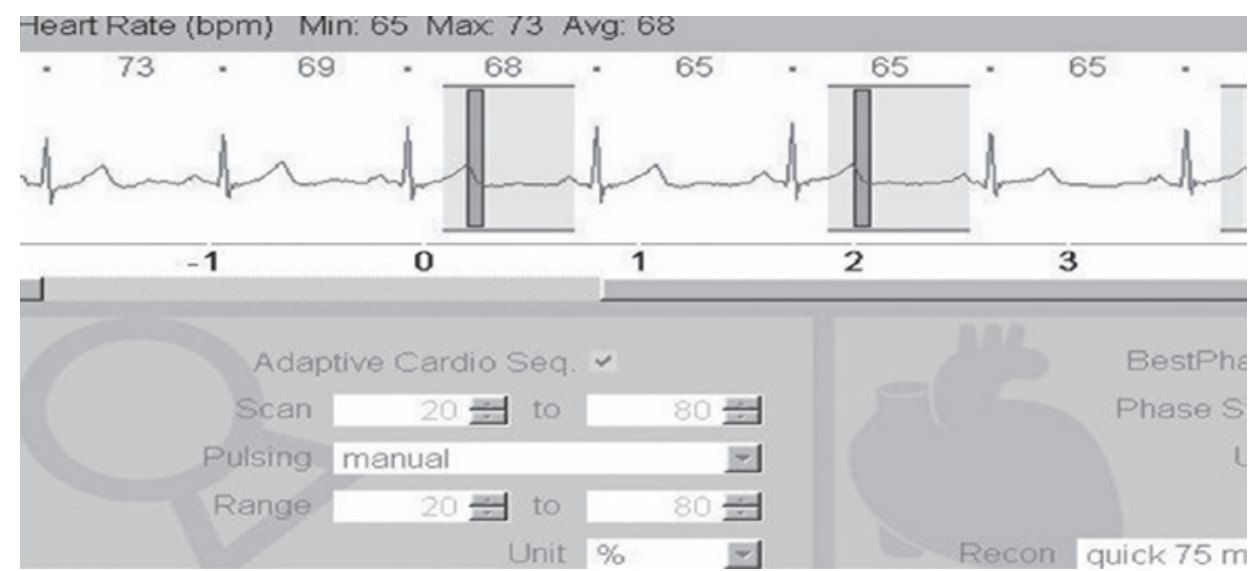

Figure 3. Scanning sketch diagram and coronary volume rendered image of group B, with a scanning range $20-80 \%$ of the R-R interval.

which reduces the rate of scan overlap and is therefore able to significantly reduce the necessary radiation dose. However, the radiation dose for patients with a high BMI remains greater compared with patients with a normal BMI, despite the use of routine sequence scanning.

ECG-ATCM scanning is based on the average heart rate prior to the scan, which is used to select a time window in the optimum phase of the cardiac cycle for full-dose exposure. The diastolic period is an important phase of the cardiac cycle and the tube current was full during this period. In addition, tube current was reduced during the systole period and decreased for the remaining period of the scan, with $20 \%$ of the full-dose exposure administered in the other heart regions $(16,17)$. Araoz et al (18) previously reported that the scanning range 
of dual-source CT is $65-70 \%$ of the R-R interval for a heart rate $\leq 70 \mathrm{bpm}$ and $35-40 \%$ of the R-R interval for a heart rate $>70 \mathrm{bpm}$.

In the sequence scanning of the present study, for group A patients with a high BMI and a heart rate $\leq 70 \mathrm{bpm}$, the scanning range was $20-80 \%$ of the R-R interval, and patients received full-dose radiation for $60-80 \%$ of the $\mathrm{R}-\mathrm{R}$ interval. For group A patients with heart rates $>70 \mathrm{bpm}$, the scanning range was $20-80 \% \mathrm{R}-\mathrm{R}$ of the interval, with patients receiving a full X-ray dose for $35-55 \%$ of the R-R interval and a $20 \%$ $\mathrm{X}$-ray dose for the remainder of the scanning period (20-35\% and $55-80 \%$ of the R-R interval). In this manner, the required radiation dose may be further reduced. There are drawbacks to this prospective scanning technology, as the data was obtained from only part of the full heartbeat cycle. It cannot undergo ECG editing and cannot evaluate the heart function, particularly in cases of patients with an irregular heartbeat, which may result in a failed scan.

In conclusion, dual-source $\mathrm{CT}$ is able to produce high quality coronary artery images $(19,20)$, while exposing patients to relatively low radiation doses. ATCM and alternative technologies, including pitch-heart rate automatic matching, facilitate significant reductions in the radiation dose of CTCA examination compared with standard CTCA. Furthermore, the use of ECG-ATCM in prospective sequence scanning permits the selection of different total dose exposure windows based on patient heart rate. This selectivity may significantly reduce the required radiation dose in overweight patients. In order to obtain satisfactory image quality, doctors and imaging technicians require an extensive understanding of the hazards of ionizing radiation and should aim to achieve low-dose scanning to reduce the exposure of patients to hazardous radiation.

\section{References}

1. Shah DJ, Sachs RK and Wilson DJ: Radiation-induced cancer: a modern view. Br J Radiol 85: e1166-e1173, 2012.

2. Sodickson A, Baeyens PF, Andriole KP, et al: Recurrent CT, cumulative radiation exposure, and associated radiation-induced cancer risks from CT of adults. Radiology 251: 175-184, 2009.

3. Husmann L, Valeta I, Gaemperli O, et al: Feasibility of low-dose coronary $\mathrm{CT}$ angiography: first experience with prospective ECG-gating. Eur Heart J 29: 191-197, 2008.

4. Buechel RR, Husmann L, Herzog BA, et al: Low-dose computed tomography coronary angiography with prospective electrocardiogram triggering: feasibility in a large population. J Am Coll Cardiol 57: 332-336, 2011.

5. Achenbach S, Goroll T, Seltmann M, et al: Detection of coronary artery stenoses by low-dose, prospectively ECG-triggered high-pitch spiral coronary CT angiography. JACC Cardiovasc Imaging 4: 328-337, 2011.

6. Abada HT, Larchez C, Daoud B, et al: MDCT of the coronary arteries: feasibility of low-dose CT with ECG-pulsed tube current modulation to reduce radiation dose. AJR Am J Roentgenol 186 (Suppl 2): S387-S390, 2006.
7. Austen WG, Edwards JE, Frye RL, et al: A reporting system on patients evaluated for coronary artery disease. Report of the Ad Hoc Committee for Grading of Coronary Artery Disease, Council on Cardiovascular Surgery, American Heart Association. Circulation 51 (4 Suppl): 5-40, 1975.

8. Alkadhia H, Stolzmanna P, Scheffel H, et al: Radiation dose of cardiac dual-source CT: the effect of tailoring the protocol to patient-specific parameters. Eur J Radiol 68: 385-391, 2008.

9. Nickoloff EL and Alderson PO: Radiation exposures to patients from CT: reality, public perception, and policy. AJR Am J Roentgenol 177: 285-287, 2001.

10. Budoff MJ, Achenbach S, Blumenthal RS, et al; American Heart Association Committee on Cardiovascular Imaging and Intervention; American Heart Association Council on Cardiovascular Radiology and Intervention; American Heart Association Committee on Cardiac Imaging, Council on Clinical Cardiology : Assessment of coronary artery disease by cardiac computed tomography: A scientific statement from the American Heart Association Committee on Cardiovascular Imaging and Intervention, Council on Cardiovascular Radiology and Intervention, and Committee on Cardiac Imaging, Council on Clinical Cardiology. Circulation 114: 1761-1791, 2006.

11. Davis F, Il'yasova D, Rankin K, et al: Medical diagnostic radiation exposures and risk of gliomas. Radiat Res 175: 790-796, 2011

12. Budoff MJ, Achenbach S, Blumenthal RS, et al; American Heart Association Committee on Cardiovascular Imaging and Intervention; American Heart Association Council on Cardiovascular Radiology and Intervention; American Heart Association Committee on Cardiac Imaging, Council on Clinical Cardiology: Assessment of coronary artery disease by cardiac computed tomography: a scientific statement from the American Heart Association Committee on Cardiovascular Imaging and Intervention, Council on Cardiovascular Radiology and Intervention, and Committee on Cardiac Imaging, Council on Clinical Cardiology. Circulation 114: 1761-1791, 2006.

13. Slovis TL: CT and computed radiography: the pictures are great, but is the radiation dose greater than required? AJR Am J Roentgenol 179: 39-41, 2002.

14. Manowitz A, Sedlar M, Griffon M, et al: Use of BMI guidelines and individual dose tracking to minimize radiation exposure from low-dose helical chest CT scanning in a lung cancer screening program. Acad Radiol 19: 84-88, 2012.

15. Arnoldi E, Johnson TR, Rist C, et al: Adequate image quality with reduced radiation dose in prospectively triggered coronary CTA compared with retrospective techniques. Eur Radiol 19: 2147-2155, 2009.

16. Earls JP, Berman EL, Urban BA, et al: Prospectively gated transverse coronary $\mathrm{CT}$ angiography versus retrospectively gated helical technique: improved image quality and reduced radiation dose. Radiology 246: 742-753, 2008.

17. Scheffel H, Alkadhi H, Plass A, et al: Accuracy of dual-source CT coronary angiography: First experience in a high pre-test probability population without heart rate control. Eur Radiol 16: 2739-2747, 2006.

18. Araoz PA, Kirsch J, Primak AN, et al: Optimal image reconstruction phase at low and high heart rates in dual-source CT coronary angiography. Int J Cardiovasc Imaging 25: 837-845, 2009.

19. Hamon M, Morello R, Riddell JW and Hamon M: Coronary arteries: diagnostic performance of 16 - versus 64 -section spiralCT compared with invasive coronary angiography - meta-analysis. Radiology 245: 720-731, 2007.

20. Paul JF, Amato A and Rohnean A: Low-dose coronary-CT angiography using step and shoot at any heart rate: comparison of image quality at systole for high heart rate and diastole for low heart rate with a 128-slice dual-source machine. Int J Cardiovasc Imaging 29: 651-657, 2013. 